\title{
Quantum Graphs: A model for Quantum Chaos
}

\author{
Tsampikos Kottos ${ }^{\mathrm{a}}$ and Holger Schanz ${ }^{\mathrm{a}, \mathrm{b}}$ \\ a Max-Planck-Institut für Strömungsforscung, \\ 37073 Göttingen, Germany \\ b Institut für Nichtlineare Dynamik, Georg-August-Universität, \\ 37073 Göttingen, Germany
}

\begin{abstract}
We study the statistical properties of the scattering matrix associated with generic quantum graphs. The scattering matrix is the quantum analogue of the classical evolution operator on the graph. For the energy-averaged spectral form factor of the scattering matrix we have recently derived an exact combinatorial expression. It is based on a sum over families of periodic orbits which so far could only be performed in special graphs. Here we present a simple algorithm implementing this summation for any graph. Our results are in excellent agreement with direct numerical simulations for various graphs. Moreover we extend our previous notion of an ensemble of graphs by considering ensemble averages over random boundary conditions imposed at the vertices. We show numerically that the corresponding form factor follows the predictions of random-matrix theory when the number of vertices is large - even when all bond lengths are degenerate. The corresponding combinatorial sum has a structure similar to the one obtained previously by performing an energy average under the assumption of incommensurate bond lengths.
\end{abstract}

PACS: 05.45.Mt, 03.65.Sq

Keywords: quantum chaos; random-matrix theory; periodic-orbit theory

\section{Introduction}

Quantum graphs have recently attracted a lot of interest [1] [ A review paper containing a list of references to previous work can be found in 11. The attention is due to the fact that quantum graphs can be viewed as typical and simple examples for the large class of systems in which classically chaotic dynamics implies universal spectral correlations in the semiclassical limit [9, 10]. Up to now we have only a very limited understanding of the reasons for this universality. In a semiclassical approach to this problem the main stumbling block is the intricate interference between the contributions of (exponentially many) periodic orbits [11,12]. Using quantum graphs as model systems it is possible to pinpoint and isolate this central problem. In graphs, an exact

\footnotetext{
${ }^{1}$ To be published in Physica E, special issue "Dynamics in Complex Systems".
}

trace formula exists which is based on the periodic orbits of a mixing classical dynamical system [1, 13. Moreover the orbits can be specified by a finite symbolic code with Markovian grammar. Based on these simplifications it is possible to rewrite the spectral form factor or other two-point correlation functions in terms of a combinatorial problem [2]. This combinatorial problem has been solved with promising results for selected graphs: It was shown that the form factor, ensemble averaged over graphs with a single nontrivial vertex and two attached bonds (2-Hydra) coincides exactly with the random-matrix result for the $2 \times 2$ CUE [2]. In [6] the short-time expansion of the form factor for $\mathrm{N}$-Hydra graphs (i. e. one central node with $N$ bonds attached) was computed in the limit $N \rightarrow \infty$, and in [7] a periodic-orbit sum was used to prove Anderson localization in an infinite chain graph with ran- 
domized bond lengths. In [8] the form factor of binary graphs was shown to approach the randommatrix prediction when the number of vertices increases. In the present paper we develop a general method to implement the combinatorial sum on a computer. Our results are always compared with direct numerical simulations.

Most of the studies on quantum graphs presented up to now were done with a fixed set of vertex boundary conditions at the vertices. In this case, one of the main premises to find spectra following the random-matrix predictions is that all bond lengths of the graph are rationally independent. In contrast, we will consider here also statistical properties of graph spectra averaged over the set of boundary conditions. We show that in this case the form factor is in agreement with the random-matrix theory (RMT) predictions even when all bond lengths are degenerate. Moreover, we show that one can express the form factor averaged over the vertex-scattering matrices as a combinatorial sum over families of orbits. This sum has the same structure as obtained in [2] by performing a spectral average.

This paper is structured in the following way. In the following Section 2, the main definitions and properties of quantum graphs are given. We concentrate on the unitary bond-scattering matrix $S_{B}$ which can be interpreted as a quantum evolution operator on the graph. Section 3 deals with the corresponding classical dynamical system. In Section 4, the statistical properties of the eigenphase spectrum of the bond-scattering matrix $S_{B}$ are analyzed and related to the periodic orbits of the classical dynamics. Finally, our conclusions are summarized in Section 5.

\section{Quantum Graphs: Basic Facts}

We start with a presentation and discussion of the Schrödinger operator for graphs. Graphs consist of $V$ vertices connected by $B$ bonds. The valency $v_{i}$ of a vertex $i$ is the number of bonds meeting at that vertex. The graph is called $v$-regular if all the vertices have the same valency $v$. When the vertices $i$ and $j$ are connected, we denote the connecting bond by $b=(i, j)$. The same bond can also be referred to as $\vec{b} \equiv(\operatorname{Min}(i, j), \operatorname{Max}(i, j))$ or $\overleftarrow{b} \equiv(\operatorname{Max}(i, j), \operatorname{Min}(i, j))$ whenever we need to assign a direction to the bond. A bond with coinciding endpoints is called a loop. Finally, a graph is called bipartite if the vertices can be divided into two disjoint groups such that any vertices belonging to the same group are not connected.

Associated to every graph is its connectivity (adjacency) matrix $C_{i, j}$. It is a square matrix of size $V$ whose matrix elements $C_{i, j}$ are given in the following way

$$
C_{i, j}=C_{j, i}=\left\{\begin{array}{l}
1 \text { if } i, j \text { are connected } \\
0 \text { otherwise }
\end{array}\right\} .
$$

For graphs without loops the diagonal elements of $C$ are zero. The connectivity matrix of connected graphs cannot be written as a block diagonal matrix. The valency of a vertex is given in terms of the connectivity matrix, by $v_{i}=\sum_{j=1}^{V} C_{i, j}$ and the total number of undirected bonds is $B=\frac{1}{2} \sum_{i, j=1}^{V} C_{i, j}$.

For the quantum description we assign to each bond $b=(i, j)$ a coordinate $x_{i, j}$ which indicates the position along the bond. $x_{i, j}$ takes the value 0 at the vertex $i$ and the value $L_{i, j} \equiv L_{j, i}$ at the vertex $j$ while $x_{j, i}$ is zero at $j$ and $L_{i, j}$ at $i$. We have thus defined the length matrix $L_{i, j}$ with matrix elements different from zero, whenever $C_{i, j} \neq 0$ and $L_{i, j}=L_{j, i}$ for $b=$ $1, \ldots, B$. The wave function $\Psi$ contains $B$ components $\Psi_{b_{1}}\left(x_{b_{1}}\right), \Psi_{b_{2}}\left(x_{b_{2}}\right), \ldots, \Psi_{b_{B}}\left(x_{b_{B}}\right)$ where the set $\left\{b_{i}\right\}_{i=1}^{B}$ consists of $B$ different undirected bonds.

The Schrödinger operator (with $\hbar=2 m=1$ ) is defined on a graph in the following way: On each bond $b$, the component $\Psi_{b}$ of the total wave function $\Psi$ is a solution of the one-dimensional equation

$$
\left(-\mathrm{i} \frac{\mathrm{d}}{\mathrm{d} x}-A_{b}\right)^{2} \Psi_{b}(x)=k^{2} \Psi_{b}(x) .
$$

We included a "magnetic vector potential" $A_{b}$ (with $\Re e\left(A_{b}\right) \neq 0$ and $A_{\vec{b}}=-A_{b}^{\leftarrow}$ ) which breaks the time reversal symmetry. In most applications we shall assume that all the $A_{b}$ 's are equal and the bond index will be dropped. On each of the bonds, the general solution of (11) is a superposition of two counter propagating waves

$\Psi_{b=(i, j)}=a_{i, j} \mathrm{e}^{\mathrm{i}\left(k+A_{i, j}\right) x_{i, j}}+a_{j, i} \mathrm{e}^{\mathrm{i}\left(k+A_{j, i}\right) x_{j, i}}$ 
The coefficients $a_{i, j}$ form a vector $\mathbf{a} \equiv\left(a_{\vec{b}_{1}}\right.$, $\left.\cdots, a_{\vec{b}_{B}}, a_{b_{1}}^{\leftarrow}, \cdots, a_{b_{B}}^{\leftarrow}\right)^{T}$ of complex numbers which uniquely determines an element in a $2 B$-dimensional Hilbert space. This space corresponds to "free wave" solutions since we did not yet impose any conditions which the solutions of (11) have to satisfy at the vertices.

The most general boundary conditions at the vertices are given in terms of unitary $v_{j} \times v_{j}$ vertex-scattering matrices $\sigma_{l, m}^{(j)}(k)$, where $l$ and $m$ go over all the vertices which are connected to $j$. At each vertex $j$, incoming and outgoing components of the wave function are related by

$a_{j, l}=\sum_{m=1}^{v_{j}} \sigma_{l, m}^{(j)}(k) e^{\mathrm{i} k L_{j m}} a_{m, j}$,

which implies current conservation. The particular form

$\sigma_{l, m}^{(j)}=\frac{2}{v_{j}}-\delta_{l, m}$

for the vertex-scattering matrices was shown in (1) to be compatible with continuity of the wave function and current conservation at the vertices. (位) is referred to as Neumann boundary conditions.

Stationary states of the graph satisfy (3) at each vertex. These conditions can be combined into

$\mathbf{a}=S_{B}(k) \mathbf{a}$,

such that the secular equation determining the eigenenergies and the corresponding eigenfunctions of the graph is of the form [1]

$\operatorname{det}\left[I-S_{B}(k, A)\right]=0$.

Here, the unitary bond-scattering matrix

$S_{B}(k, A)=D(k ; A) T$

acting in the $2 B$-dimensional space of directed bonds has been introduced. The matrices $D$ and $T$ are given by

$$
\begin{aligned}
D_{i j, i^{\prime} j^{\prime}}(k, A) & =\delta_{i, i^{\prime}} \delta_{j, j^{\prime}} \mathrm{e}^{\mathrm{i} k L_{i j}+\mathrm{i} A_{i, j} L_{i j}} \\
T_{j i, n m} & =\delta_{n, i} C_{j, i} C_{i, m} \sigma_{j, m}^{(i)} .
\end{aligned}
$$

$T$ contains the topology of the graph and is equivalent to the complete set of vertex-scattering matrices, while $D$ contains the metric information about the bonds.

It is instructive to interpret the action of $S_{B}$ on an arbitrary graph state $\Psi$ as its time evolution over an interval corresponding to the mean bond length of the graph such that

$\mathbf{a}(n)=S_{B}^{n} \mathbf{a}(0), \quad n=0,1,2, \ldots$.

Clearly the solutions of (5) are stationary with respect to this time evolution. $n$ in (9) represents a discrete (topological) time counting the collisions of the particle with vertices of the graph.

\section{Periodic orbits and classical dynamics on graphs}

In this section we discuss the classical dynamics corresponding to the quantum evolution (9) implied by $S_{B}$. To introduce this dynamics we employ a Liouvillian approach, where a classical evolution operator assigns transition probabilities in a phase space of $2 B$ directed bonds [1]. If $\rho_{b}(t)$ denotes the probability to occupy the (directed) bond $b$ at the (discrete) topological time $t$, we can write down a Markovian Master equation of the form

$\rho_{b}(t+1)=\sum_{b^{\prime}} U_{b, b^{\prime}} \rho_{b^{\prime}}(t)$.

The classical (Frobenius-Perron) evolution operator $U$ has matrix elements

$U_{i j, n m}=\delta_{j, n} P_{i \rightarrow m}^{(j)}$

with $P_{j i \rightarrow i j^{\prime}}^{(i)}$ denoting the transition probability between the directed bonds $b=(j, i)$ and $b^{\prime}=$ $\left(i, j^{\prime}\right)$. To make the connection with the quantum description, we adopt the quantum transition probabilities, expressed as the absolute squares of matrix elements of $S_{B}$

$P_{j \rightarrow j^{\prime}}^{(i)}=\left|\sigma_{j, j^{\prime}}^{(i)}(k)\right|^{2}$.

Note that $P_{j \rightarrow j^{\prime}}^{(i)}$ and $U$ do not involve any metric information on the graph. In general, they may depend on the wave number $k$. 
The unitarity of the bond-scattering matrix $S_{B}$ guarantees $\sum_{b=1}^{2 B} U_{b, b^{\prime}}=1$ and $0 \leq U_{b, b^{\prime}} \leq 1$, so that the total probability that the particle is on any bond remains conserved during the evolution. The spectrum of $U$ is restricted to the interior of the unit circle and $\nu_{1}=1$ is always an eigenvalue with the corresponding eigenvector $|1\rangle=\frac{1}{2 B}(1,1, \ldots, 1)^{T}$. In most cases, the eigenvalue 1 is the only eigenvalue on the unit circle. Then, the evolution is ergodic since any initial density will evolve to the eigenvector $|1\rangle$ which corresponds to a uniform distribution (equilibrium). The rate at which equilibrium is approached is determined by the gap to the next largest eigenvalue. If this gap exists, the dynamics is also mixing. Graphs are one dimensional and the motion on the bonds is simple and stable. Ergodic (mixing) dynamics is generated because at each vertex a (Markovian) choice of one out of $v$ directions is made. Thus, chaos on graphs originates from the multiple connectivity of the (otherwise linear) system [1].

Despite the probabilistic nature of the classical dynamics, the concept of a classical orbit can be introduced. A classical orbit on a graph is an itinerary of successively connected directed bonds $\left(i_{1}, i_{2}\right),\left(i_{2}, i_{3}\right), \cdots$. An orbit is periodic with pe$\operatorname{riod} n$ if for all $k,\left(i_{n+k}, i_{n+k+1}\right)=\left(i_{k}, i_{k+1}\right)$. For graphs without loops or multiple bonds, the sequence of vertices $i_{1}, i_{2}, \cdots$ with $i_{m} \in[1, V]$ and $C_{i_{m}, i_{m+1}}=1$ for all $m$ represents a unique code for the orbit. This is a finite coding which is governed by a Markovian grammar provided by the connectivity matrix. In this sense, the symbolic dynamics on the graph is Bernoulli. This analogy is strengthened by further evidence: The number of $n$-PO's on the graph is $\frac{1}{n} \operatorname{tr} C^{n}$, where $C$ is the connectivity matrix. Since its largest eigenvalue $\Gamma_{c}$ is bounded between the minimum and the maximum valency i.e. $\min v_{i} \leq \Gamma_{c} \leq \max v_{i}$, periodic orbits proliferate exponentially with topological entropy $\approx \log \Gamma_{c}$.

\section{The spectral statistics of $S_{B}$}

We consider the matrix $S_{B}(k, A)$ defined in (7), (8). The spectrum consist of $2 B$ points $e^{\mathrm{i} \theta_{l}(k)}$ confined to the unit circle (eigenphases). Uni- tary matrices of this type are frequently studied since they are the quantum analogues of classical, area preserving maps. Their spectral fluctuations depend on the nature of the underlying classical dynamics 14]. The quantum analogues of classically integrable maps display Poissonian statistics while in the opposite case of classically chaotic maps, the eigenphase statistics conform with the results of RMT for Dyson's circular ensembles. To describe the spectral fluctuations of $S_{B}$ we consider the form factor

$K(n / 2 B)=\frac{1}{2 B}\left\langle\left|\operatorname{tr} S_{B}^{n}\right|^{2}\right\rangle \quad(n>0)$.

The average $\langle\ldots\rangle$ will be specified below. RMT predicts that $K(n, 2 B)$ depends on the scaled time $\tau=\frac{n}{2 B}$ only [14], and explicit expressions for the orthogonal and the unitary circular ensembles are known [15].

Using (7), (8) we expand the matrix products in $\operatorname{tr} S_{B}^{n}$ and obtain a sum of the form

$\operatorname{tr} S_{B}^{n}(k)=\sum_{p \in \mathcal{P}_{n}} \mathcal{A}_{p} \mathrm{e}^{\mathrm{i}\left(k L_{p}+A l_{p}\right)}$.

In this sum $p$ runs over all closed trajectories on the graph which are compatible with the connectivity matrix and which have the topological length $n$, i. e. they visit exactly $n$ vertices. For graphs, the concepts of closed trajectories and periodic orbits coincide, hence (14) can also be interpreted as a periodic-orbit sum. From (14) it is clear that $K(n / 2 B)=0$ as long as $n$ is smaller than the period of the shortest periodic orbit. The phase associated with an orbit is determined by its total (metric) length $L_{p}=\sum_{b \in p} L_{b}$ and by the "magnetic flux" through the orbit. The latter is given in terms of its total directed length $l_{p}$ if we assume for simplicity that the magnitude of the magnetic vector potential is constant $\left|A_{b}\right| \equiv A$. The complex amplitude of the contribution from a periodic orbit by the product of all the elements of vertex-scattering matrices encountered

$\mathcal{A}_{p}=\prod_{j=1}^{n_{p}} \sigma_{i_{j-1}, i_{j+1}}^{\left(i_{j}\right)} \equiv \prod_{[r, s, t]}\left(\sigma_{r, t}^{(s)}\right)^{n_{p}(r, s, t)}$,

i. e. for fixed boundary conditions at the vertices it is completely specified by the frequencies 


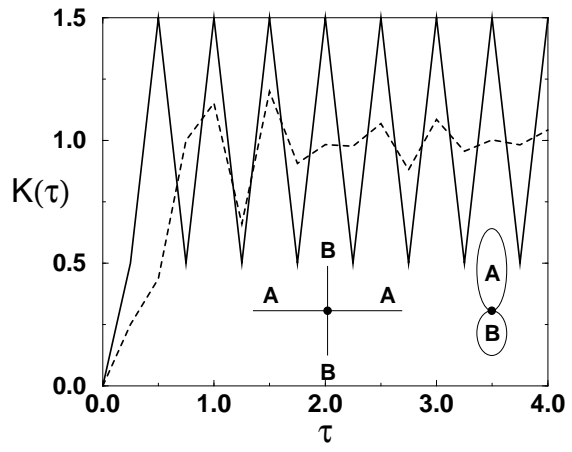

Fig. 1: Form factor of $S_{B}$ for a double-loop graph (or equivalently $S_{B}^{2}$ for a 4 -Hydra). The full line shows (18) corresponding to zero magnetic field in the loops or two pairs of degenerate bond lengths in the Hydra as shown in the inset. Dashed line (obtained from (20)): A magnetic flux threads the loops and is averaged over. This corresponds to 4 incommensurate bond lengths of the Hydra graph.

$n_{p}(r, s, t)$ of all transitions $(r, s) \rightarrow(s, t)$. Inserting (14) into the definition of the form factor we obtain a double sum over periodic orbits

$$
\begin{aligned}
& K(n / 2 B)=\frac{1}{2 B}\left\langle\sum_{p, p^{\prime} \in \mathcal{P}_{n}} \mathcal{A}_{p} \mathcal{A}_{p^{\prime}}^{*} \times\right. \\
& \left.\quad \exp \left\{\mathrm{i} k\left(L_{p}-L_{p^{\prime}}\right)+\mathrm{i} A\left(l_{p}-l_{p^{\prime}}\right)\right\}\right\rangle .
\end{aligned}
$$

This will be our starting point for the combinatorial approach presented in the following subsection.

\subsection{Energy Average}

In previous work [2, 6, ,7] the average in (16) was always taken with respect to the wave number $k$ and, if present, also over the magnetic vector potential $A$. Within the present subsection we will stick to this procedure. Provided that the bond lengths of the graph are rationally independent and that a sufficiently large interval is used for averaging, only terms with $L_{p}=L_{p^{\prime}}$ and $l_{p}=l_{p^{\prime}}$ survive. A completely equivalent result can be obtained by regarding the bond lengths $L_{b}$ of the graph as random numbers and performing an ensemble average over them.

Note that $L_{p}=L_{p^{\prime}}$ does not necessarily imply $p=p^{\prime}$ or that $p, p^{\prime}$ are related by some symmetry because there exist families $\mathcal{L}$ of distinct but isometric orbits which can be used to write the result of (16) in the form [2,6,7]

$K(n / 2 B)=\sum_{\mathcal{L} \in \mathcal{F}_{n}}\left|\sum_{p \in \mathcal{L}} \mathcal{A}_{p}\right|^{2}$.

The outer sum is over the set $\mathcal{F}_{n}$ of families, while the inner one is a coherent sum over the orbits belonging to a given family (= metric length). (17) is exact, and it represents a combinatorial problem since it does not depend anymore on metric information about the graph (the bond lengths).

Let us illustrate the application of (17) in the following simple system: We consider a Hydra graph with four arms and impose Neumann boundary conditions at the central node such that forward and backward scattering amplitudes are given according to (何) by $\sigma_{f}=1 / 2$ and $\sigma_{b}=-1 / 2$. For the moment we require that there are two pairs of identical bond lengths $L_{A}, L_{B}$ (see Fig. 1). Since Hydra is a bipartite graph, an orbit can return only after an even number of vertex-scattering events (note that the dead ends of the arms are considered as vertices). It is then convenient to replace the scattering matrix in (13) by $S_{B}^{2}$. As one can see from (14), that the form factor obtained in this way is equivalent to the one obtained from a graph which is formed by two loops of lengths $2 L_{A}$ and $2 L_{B}$ connected by a single vertex (inset of Fig. 1). Consider now the periodic orbits contributing to $K(n / 2 B)$. The length of such an orbit is given by $L=n_{A} L_{A}+n_{B} L_{B}$ where $n_{A}, n_{B}$ denotes the number of traversals of $\mathrm{A}$ and $\mathrm{B}$ loop, respectively, and $n=n_{A}+n_{B}$. If $L_{A}, L_{B}$ are rationally independent, a family of isometric orbits in (17) contains all orbits shar$\operatorname{ing} n_{A}, n_{B}$. These orbits differ, however, in their symbolic code by the order of A's and B's and by the orientation in which the loops are traversed. This is reflected in the amplitudes $\mathcal{A}_{p}$ of the orbits: If two consecutive loops AA have different orientation, the amplitude $\mathcal{A}_{p}$ contains a factor $\sigma_{b}$, while the same orientation results in $\sigma_{f}$. Different loops following each other (AB or BA) always result in a factor $\sigma_{f}$. Suppose now there is an orbit which contains in its code a sequence of the form $\cdots \mathrm{AB}_{1} \mathrm{~B}_{2} \mathrm{~B}_{s} \cdots A \cdots$. Then there will be another orbit $\cdots \mathrm{AB}_{1} \mathrm{~B}_{2}{ }_{2} \mathrm{~B}_{s} \cdots A \cdots$ which differs 
from the first one by an inversion of the orientation (denoted by the prime) of all but the first loop B. It is easy to see that the corresponding amplitudes cancel. This results in a tremendous simplification of the problem: It suffices in (17) to keep track of all trajectories for which the cancellation does not apply. These are of the form $\mathrm{A}^{n}, \mathrm{~B}^{n},(\mathrm{AB})^{n / 2}$ or $(\mathrm{BA})^{n / 2}$, the latter two existing for even $n$ only. Each orbit $\mathrm{A}^{n}$ contributes the amplitude $1 / 2^{n}$ irrespective of the orientations of the $n$ loops. On the other hand there are $2^{n}$ possibilities to prescribe these orientations and hence the total contribution from each of the families $\mathrm{A}^{n}$ and $\mathrm{B}^{n}$ is 1 . There are also $2^{n}$ trajectories of the form $(\mathrm{AB})^{n / 2}$ and $(\mathrm{BA})^{n / 2}$, respectively, each with amplitude $1 / 2^{n}$. However these orbits belong to the same family $n_{A}=n_{B}=n / 2$, and their amplitudes must be added coherently in (17). This results in the total contribution 4 from this family. After adding the contributions from the three different families and normalizing with $2 B=4$ we are left with

$K(n / 2 B)=\left\{\begin{array}{ll}1 / 2 & n=1,3, \ldots \\ 3 / 2 & n=2,4, \ldots\end{array}\right.$,

which is shown in Fig. 1 with a solid line. Neglecting the point $n=0$, the form factor simply oscillates around the value 1 - even within the Heisenberg time $\tau=n / 2 B=1$. This is reminiscent of the behavior of a Poissonian spectrum. The reason for this lack of correlations is that there exist regular sequences of eigenstates which are completely confined to one of the two loops or one pair of degenerate bonds, respectively. These states disappear when all arms of the Hydra are incommensurate, and the corresponding form factor (dashed line in Fig. 1) clearly reflects this change in the properties of the spectrum.

The arguments which led to (18) are deceivingly simple. In general, the combinatorial problem (17) is very hard and cannot be solved in closed form. Even for the 4-Hydra with incommensurate bond lengths this seems to be the case. Nevertheless exact result for finite $n$ as those shown in Fig. 1 with a dashed line can always be obtained from (17) using a computer algebra system such as Maple [17]. This will be shown in the following. To prescribe topology and boundary conditions, one should provide the time evo- lution operator $S_{B}$ according to (7) but with the phases $\mathrm{e}^{\mathrm{i} k L_{i, j}}$ left as unspecified variables. For example, the operator $S_{B}$ for the graph discussed above has the form

$$
\left[\begin{array}{cccc}
-1 / 2 \phi_{A} & 1 / 2 \phi_{A} & 1 / 2 \phi_{B} & 1 / 2 \phi_{B} \\
1 / 2 \phi_{A} & -1 / 2 \phi_{A} & 1 / 2 \phi_{B} & 1 / 2 \phi_{B} \\
1 / 2 \phi_{A} & 1 / 2 \phi_{A} & -1 / 2 \phi_{B} & 1 / 2 \phi_{B} \\
1 / 2 \phi_{A} & 1 / 2 \phi_{A} & 1 / 2 \phi_{B} & -1 / 2 \phi_{B}
\end{array}\right]
$$

where $\phi_{A / B}=\mathrm{e}^{\mathrm{i} 2 k L_{A / B}}$. In the general case there will be as many phases $\phi_{i}$ as there are incommensurate lengths in the graph. The quantity $\operatorname{tr} S_{B}^{n}$ can now be represented as a multivariate polynomial of degree $n$ in the variables $\phi_{i}$, i. e.

$\operatorname{tr} S_{B}^{n}=\sum_{\mathcal{P}_{n}} c_{\mathcal{P}} \phi_{1}^{p_{1}} \phi_{2}^{p_{2}} \ldots$,

where $\mathcal{P}_{n}$ runs over all partitions of $n$ into nonnegative integers $n=p_{1}+p_{2}+\ldots$ The form factor is then simply given as

$K(n / 2 B)=\sum_{\mathcal{P}_{n}}\left|c_{\mathcal{P}}\right|^{2}$.

The task of finding the coefficients $c_{\mathcal{P}}$ can be expressed in Maple with standard functions, such that after the initialization of $S_{B}$ the following four simple lines represent a completely general algorithm for the exact computation of the form factor of an arbitrary graph:

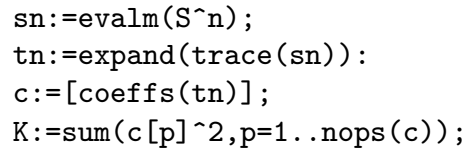

In practice, however, the computation is restricted to the first few $n$ since the numerical effort grows exponentially fast. In Fig. 2 we compare the results of (20) with direct numerical averages for regular (fully connected) graphs with $V=4$ and $V=5$ vertices with and without magnetic field breaking the time-reversal symmetry. The results agree indeed to a high precision. Although this could be regarded merely as an additional confirmation of the numerical procedures used in 11, we see the main merit of (20) in being a very useful tool for trying to find the solution of (17) in closed form. 


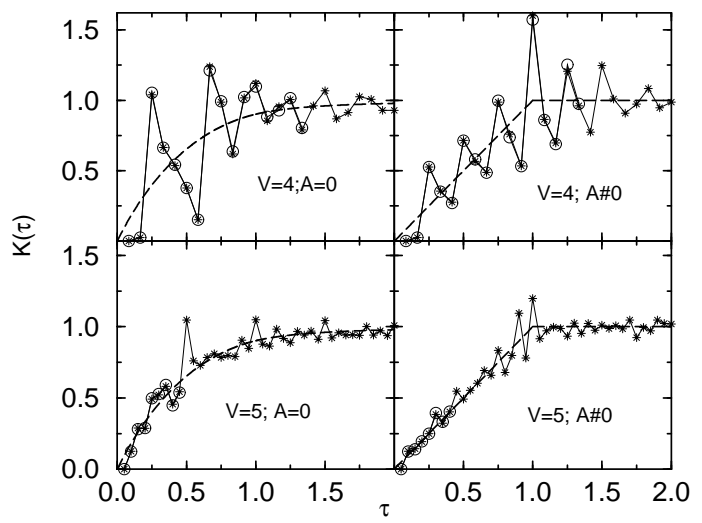

FIG. 2. Form factor of $S_{B}$ for regular graphs with $V=4$ vertices (top) and $V=5$ vertices (bottom). In the right panels an additional magnetic field destroyed timereversal symmetry. Circles: exact results obtained from (17). Stars: numerical average over 2,000 values of $k$. The solid line is to guide the eye. The prediction of the appropriate random matrix ensemble are shown with dashed lines.

\subsection{Ensemble Average over the Boundary Conditions at Vertices}

In the present section we generate an ensemble of graphs by randomizing the vertex-scattering matrices $\sigma^{(i)}$. The length matrix $L$ which contains the lengths of the bonds and the connectivity matrix (topology of the graph) are kept constant. Moreover all the bond lengths are equal $L_{b}=1$. We computed the form factor for fully connected graphs. Our results for values of $V=10,15,20$ are presented in Fig. 3. The RMT two-point form factor 15] is also displayed in Fig. 3 for comparison. The results show quite a good agreement with the predictions of RMT for the circular ensembles. Moreover, one can see that as the number of vertices $V$ increases, the agreement with RMT becomes progressively better. The deviations from the smooth curves are not statistical, and cannot be ironed out by further averaging. Rather, they are due to the fact that the graph is a dynamical system which cannot be described by RMT in all detail.

In the remainder of this subsection, we would like to point out the possibility for turning (16)

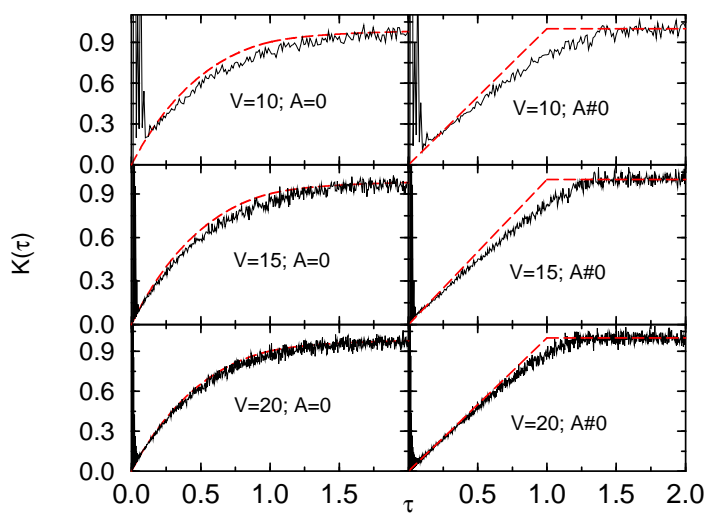

Fig. 3: The form factor of the eigenphase spectrum of $S_{B}$ for fully connected graphs with $V=10,15,20$. Bold dashed lines are the expectations for the COE and CUE expressions. The data are averaged over realizations of the $\sigma$ 's, as explained in the text. All the bond lengths are kept constant and equal to $L_{i . j}=1$.

into a combinatorial problem using the ensembleaverage over $\sigma^{(i)}$. We assume that $\sigma^{(i)} i=1, \ldots V$ are independent random matrices chosen from the CUE. In this case, the averaging in (16) factorizes with respect to the vertices and we find

$$
\begin{aligned}
\left\langle\mathcal{A}_{p} \mathcal{A}_{p \prime}^{*}\right\rangle=\prod_{s}\left\langle\prod_{[r, t]}\left(\sigma_{r, t}^{(s)}\right)^{n_{p}(r, s, t)} \times\right. \\
\left.\left(\sigma_{r, t}^{(s) *}\right)^{n_{p^{\prime}}(r, s, t)}\right\rangle
\end{aligned}
$$

Averages over the CUE of this type have been computed in the literature, see e. g. [16]. The result is of the form

$$
\begin{array}{r}
\left\langle U_{a_{1}, b_{1}} \ldots U_{a_{n}, b_{n}} \times U_{a_{1}, b_{1}} \ldots U_{\alpha_{n}, \beta_{n}}\right\rangle_{C U E}= \\
\sum_{P, P^{\prime}} C_{P^{-1} P^{\prime}} \prod_{j=1}^{n} \delta_{a_{j}, \alpha_{P(j)}} \delta_{b_{j}, \beta_{P^{\prime}(j)}}, \quad(22)
\end{array}
$$

where $P, P^{\prime}$ run over the permutations of $1, \ldots, n$ and $C_{P^{-1} P^{\prime}}$ are some constants which can be obtained from a recursion relation. Applied to (21) this implies, that the contribution from a pair of orbits $p, p^{\prime}$ vanishes unless for all $s, t$ $\sum_{r} n_{p}(r, s, t)=\sum_{r} n_{p^{\prime}}(r, s, t) \sum_{r} n_{p}(r, s, t)=$ $\sum_{r} n_{p^{\prime}}(r, s, t)$, i. e. unless the frequencies of traversals of any directed bond $s \rightarrow t$ coincide for $p$ 
and $p^{\prime}$. For the case of a graph with time-reversal symmetry, i. e. when the vertex-scattering matrices are taken at random from the COE, a similar result ensures that only those pairs of orbits survive the averaging in (21) which agree in the traversals of all undirected bonds. This means that the family structure underlying the set of periodic orbits is exactly the same as in the case of a graph with incommensurate bond lengths but fixed boundary conditions: The families are formed by those orbits which differ only in the time order of the traversed bonds. Substituting this result into (16) we see that all oscillating phases containing metric information on the graph drop independent of the precise values of the bond lengths and we are indeed left with a combinatorial problem.

\section{Conclusions}

In this paper we have tried to contribute to the understanding of the statistical properties of the unitary quantum time evolution operator derived from quantum graphs. This problem is relevant since it is a paradigm for the as yet unanswered question precisely under which conditions the quantum analogues of classically chaotic systems are universal and follow the random-matrix predictions. Fully connected quantum graphs show this universality when the number of bonds becomes large. In extension to previous work we have demonstrated this result in the case where an ensemble of graphs is introduced by randomizing the boundary conditions at the vertices. The corresponding ensemble average can replace the previously considered spectral average, and the RMT results are in this case approached even when all the bond lengths are incommensurate. We have the hope that this kind of ensemble average might provide an easier access to an analytical treatment of the spectral properties of graphs. One possible approach of this goal is the use of combinatorial methods to perform the periodicorbit sums related to spectral two-point correlations.

\section{Acknowledgements}

We would like to express our gratitude to Prof. Uzy Smilansky to whom we owe our interest in the subject and who contributed a great deal to most results discussed in this paper.

\section{REFERENCES}

1. T. Kottos and U. Smilansky, Phys. Rev. Lett. 79, 4794 (1997). Annals of Physics 274, 76 (1999).

2. H. Schanz and U. Smilansky, to be published in the Proceedings of the Australian Summer School on Quantum Chaos and Mesoscopics, Canberra, Australia, 1999.

3. T. Kottos and U. Smilansky, chao-dyn/ 9906008.

4. F. Barra and P. Gaspard, preprint (1999).

5. E. Akkermans et al., cond-mat/9911183.

6. G. Berkolaiko and J. Keating, J. Phys. A32, 7827 (1999).

7. H. Schanz and U. Smilansky, in print for Phys. Rev. Lett.

8. G. Tanner, hlin.CD/0001025.

9. O. Bohigas, M. J. Giannoni, C. Schmit, Phys. Rev. Lett. 52, 1-4 (1984).

10. M. V. Berry, Proc. Royal Soc. London A 400, 229 (1985).

11. N. Argaman et al., Phys. Rev. Lett. 71, 4326 (1993).

12. D. Cohen, H. Primack, and U. Smilansky, Annals of Physics 264, 108-170, (1998).

13. Jean-Pierre Roth, in: Lectures Notes in Mathematics: Theorie du Potentiel, A. Dold and B. Eckmann, eds. (Springer-Verlag) 521-539.

14. U. Smilansky, in Proc. 1989 Les Houches Summer School on Chaos and Quantum Physics, M.-J. Giannoni et.al., eds. (North-Holland) 371-441.

15. M. L. Mehta, Random Matrices and the Statistical Theory of Energy Levels, Academic, New York (1990).

16. P. W. Brouwer and C. W. J. Beenakker, J. Math. Phys. 37, 4904-4934 (1996). See also references therein.

17. M. Kofler, Maple : an introduction and reference, Addison-Wesley, Harlow (1997). 